\title{
Clinicians tend to overestimate improvements in asthma control: an unexpected observation
}

\author{
Elizabeth F. Juniper ${ }^{\mathrm{a}, *}$, Anoop Chauhan ${ }^{\mathrm{b}}$, Edmund Neville ${ }^{\mathrm{b}}$, Anwesh \\ Chatterjee $^{\mathrm{b}}$, Klas Svensson ${ }^{c}$, Ann-Christin Mörk ${ }^{c}$, Elisabeth Ståhl ${ }^{c}$
}

\author{
a McMaster University, Hamilton, Canada \\ b St. Mary' Hospital, Portsmouth, UK \\ ${ }^{c}$ AstraZeneca R\&D, Lund, Sweden
}

Received 4 March 2004; accepted 26 April 2004

\section{KEYWORDS \\ Asthma; \\ Measurement; \\ Questionnaires; \\ Clinical practice}

\section{Introduction}

A well established method for estimating the minimal important difference (MID) of a health-related

\footnotetext{
* Corresponding author. Present address: 20 Marcuse Fields, Bosham, West Sussex, P018 8NA, England.

Tel.: +44 1243 572124; fax: +44 1243573680 .

E-mail address: juniper@qoltech.co.uk (E.F. Juniper).
}

\begin{abstract}
Summary
Aim: The original purpose of this study was to determine the Minimal Important Difference for the Asthma Control Questionnaire (ACQ) but an unexpected tendency of clinicians to overestimate improvements in asthma control thwarted the endeavour. We describe the observed clinician bias and discuss its implications for clinical practice and research.

Methods: Ninety-four adults with inadequately controlled asthma received a full clinical consultation with one of nine asthma specialists. Medications were adjusted according to clinical needs. Four weeks later the same clinician estimated change in asthma control on a 15 -point scale $(-7=$ a very great deal worse, $0=$ no change, +7 a very great deal better). All patients completed the ACQ before each consultation but responses were not shown to the clinician. C I C
Results: Clinicians consistently recorded that patients improved more than their change in ACQ scores suggested $(p=0.018)$.

Conclusion: Clinicians should be aware of potential biases that may occur when estimating change in asthma control compared with measuring absolute status at each visit.

( 2004 General Practice Airways Group. Published by Elsevier Ltd. All rights reserved.
\end{abstract}

quality of life questionnaire is to ask patients to complete the questionnaire on two occasions and on the second occasion to ask them whether there has been any change in their quality of life since the first visit [1]. Data from patients who indicate that they have had a small but important change are used to determine the MID. To determine the MID for the clinically-based Asthma Control Questionnaire (ACQ) [2], patients completed the ACQ at 
two consecutive clinic visits and asthma clinicians, blinded to the ACQ data, estimated whether there had been any change in patients' asthma control between the two visits.

\section{Methods}

\section{Patients}

Ninety four adults (17-70 years), who were attending an asthma clinic for the routine management of their asthma, participated in the study. They were required to have inadequately controlled asthma ( $A C Q>1.0)$ and were not permitted to have other medical conditions whose symptoms might be confused with those of asthma. All patients signed a consent that had been approved by the Isle of Wight, Portsmouth and South-East Hampshire Local Research Ethics Committee.

\section{Clinicians}

Nine clinicians (consultants $(n=2)$, registrars $(n=6)$ and an asthma nurse specialist) from the Respiratory Centre at St. Mary's Hospital, Portsmouth, UK participated in the study. They all had extensive experience in the diagnosis and treatment of asthma, a thorough understanding of the concept of 'asthma control', based on international guidelines [2], and a good grasp of the concept of the MID.

\section{Study Design}

This was a 5-week observational study in which patients were seen in the clinic on enrollment and after 1 and 5 weeks. At the first visit patients completed the informed consent and were trained to use a peak flow meter. For one week before each subsequent visit ( 1 and 5 weeks), patients made pre-bronchodilator morning and evening measurements of peak expiratory flow (PEF). At the 1 and 5 week visits, patients completed the ACQ before any discussion with the clinician or the study co-ordinator/clinic nurse. At each clinic visit, spirometry was measured before and 20 minutes after bronchodilator.

\section{Clinical Assessment}

At weeks 1 and 5 , patients were seen by a clinician (the same clinician at both visits). On both occasions, the clinician assessed asthma control using spirometry, morning and evening PEFs during the previous week, and a conventional consultation, but was blinded to the patient's ACQ responses.
At week 1, patients were given a treatment plan (it was suggested that patients with inadequately controlled asthma should have their asthma medications increased). At week 5 , the clinician scored change in asthma control between weeks 1 and 5 on a transition rating questionnaire.

\section{Outcome Measures}

\section{Asthma Control Questionnaire}

The Asthma Control Questionnaire was developed and validated to measure the goals of asthma management as defined by international guidelines (minimisation of symptoms and activity limitation, airway narrowing and rescue bronchodilator use) [2]. It has 7 questions, five of which concern nighttime and daytime symptoms and activity limitation, one about daily rescue bronchodilator use and one about $\mathrm{FEV}_{1} \%$ predicted pre-bronchodilator. The response to all 7 questions is on a 7 -point scale and patients are asked to recall their experiences during the previous week. The items are equally weighted and the ACQ score is the mean of the 7 items and therefore between 0 (well controlled) and 6 (extremely poorly controlled). The ACQ has high reliability (ICC $=0.90$ ) and it is very responsive to change in asthma control $(p<0.0001$ : responsiveness index $=1.35$ ). Evidence of strong crosssectional and longitudinal validity has been shown by correlations between the ACQ and other measures of asthma health status being very close to a priori predictions.

\section{Clinician Transition Rating Questionnaire}

This single item questionnaire asked clinicians: ceplease review your clinic notes, / peak flow rates and spirometry from this visit and the previous one. Has there been any change in the control of the patient's asthma?" Clinicians responded using a 15 point scale $(+7=$ very much better, $0=$ no change and $-7=$ very much worse). The minimal important difference (MID) was defined as 'the minimal change in score which clinicians consider to be clinically important and which would mandate, in the absence of troublesome side effects and undue cost, a change in the patient's management'. In this study, clinicians were told that changes of $-2,-3$, +2 and +3 on the transition rating questionnaire represented the minimal important difference.

\section{Inter-rater Reliability}

Sixteen patients were assessed by two clinicians at each visit who made independent estimates of change in asthma control. 


\section{Analysis}

Patients were grouped according to transition rating scores $(+7$ and $+6=$ large improvement, +5 and $+4=$ moderate improvement, +3 and +2 , minimal important improvement; $+1,0$ and $-1=$ no change, -2 and $-3=$ minimal important deterioration, -4 and $-5=$ moderate deterioration, -6 and -7 = large deterioration) [1]. Mean change in ACQ scores between weeks 1 and 5 were calculated for patients in each of the groups. Differences were examined using an unpaired t-test. Inter-rater reliability was examined using an intraclass correlation coefficient.

\section{Results}

Seventy-three patients completed the study. There were 30 males and 43 females with an average age of 51.1 years (SD 14.3 years) and $\mathrm{FEV}_{1} \%$ pred. of 74.4 (SD 26.2). At enrollment, one patient used no asthma medication; 5 patients used a short-acting $\beta_{2}$-agonist (SAB) either alone $(n=1)$ or with a longacting $\beta_{2}$-agonist (LAB) plus either ipratropium bromide $(n=1)$, theophylline $(n=1)$, montelukast $(n$ $=1$ ) or an oral steroid $(n=1) ; 20$ patients used an inhaled steroid plus a $S A B ; 49$ patients took an inhaled steroid plus both a SAB and a LAB of whom 29 used one or more of the following: theophylline ( $n$ $=12$ ), ipratropium bromide $(n=11)$, montelukast ( $n$ $=12$ ) and oral steroid $(n=9)$. Twenty-one patients failed to return to the clinic after either the first or second visit. They had similar baseline asthma control to those who completed the study but tended to be younger (both men and women).

Table 1 shows the meanchange in ACQ scores between weeks 1 and 5 for each transition rating category. It had been anticipated that patients in the no-change transition rating group would show minimal change in ACQ scores and that improvements and deteriorations would be evenly distributed about this group. However, there was a significant shift to the left $(p=0.018)$ with patients in the minimally important improvement group $(+2$
Table 2 Consistency with which clinicians rated change in asthma control.

\begin{tabular}{ccr}
$\begin{array}{c}\text { Clinician Transition } \\
\text { Rating of Change }\end{array}$ & & $\begin{array}{l}\text { Change in } \\
\text { ACQ }\end{array}$ \\
\hline Rater 1 & Rater 2 & \begin{tabular}{l} 
Score \\
\hline+4
\end{tabular} \\
+5 & +4 & 0.57 \\
+3 & +5 & 0.28 \\
+3 & +4 & -0.43 \\
+5 & +2 & 0.72 \\
+1 & +1 & 0.14 \\
+2 & +1 & -0.71 \\
+3 & +5 & 0.00 \\
+4 & +4 & 0.15 \\
-3 & -4 & 0.43 \\
+4 & +5 & -0.43 \\
+1 & +3 & 1.28 \\
+5 & +4 & -0.14 \\
+2 & +2 & 1.29 \\
-4 & -3 & 0.71 \\
+1 & +2 & -1.00 \\
\hline
\end{tabular}

and +3 ) showing the least change in ACQ scores $(0.04 \pm 0.52)$ (Table 1$)$. In other words, there was a tendency for clinicians to both overestimate improvements and underestimate deteriorations. This bias tended to be very slightly greater in men than women but the sample size was too small to make a meaningful test for significance. In the 16 patients seen by two clinicians at each visit, interclinician reliability was high (ICC $=0.88$ ) (Table 2 ). In addition, the bias occurred consistently in all 9 clinicians.

\section{Discussion}

Estimation of the MID of the ACQ could not be done using this data set as it requires an even distribution about a central 'no change' group. The observed bias was totally unexpected and large enough to be of clinical concern (a value of 0.5 on the 7-point scale having subsequently been established as the MID of the ACQ using alternative methods $[3,4]$ ). Patients who recorded the least change in their

Table 1 MID for ACQ (Mean \pm standard deviation).

\begin{tabular}{|c|c|c|c|c|c|c|c|}
\hline & \multicolumn{7}{|c|}{ Clinician Transition Rating of Change } \\
\hline & \multicolumn{3}{|c|}{ Better } & \multirow{2}{*}{$\begin{array}{c}\text { Same } \\
+1,0,-1\end{array}$} & \multicolumn{3}{|c|}{ Worse } \\
\hline & $+7,+6$ & $+5,+4$ & $\begin{array}{c}+3,+2 \\
\text { MID }\end{array}$ & & $\begin{array}{c}-2,-3 \\
\text { MID }\end{array}$ & $-4,-5$ & $-6,-7$ \\
\hline $\begin{array}{l}\text { Change in mean } \\
\text { ACQ score between weeks } 1 \text { and } 5\end{array}$ & $0.29(1)$ & $0.60 \pm 0.77$ & $0.04 \pm 0.52$ & $-0.10 \pm 0.39$ & $-0.48 \pm 0.46$ & $-0.26 \pm 0.83$ & -2.14 \\
\hline (number of patients) & (1) & $(21)$ & $(18)$ & (17) & (9) & (6) & (1) \\
\hline
\end{tabular}


ACQ scores were considered by their clinicians to have had clinically important improvements in their asthma control ( +2 and +3 on the transition scale). This bias was very consistent across all clinicians and there was a high inter-clinician reliability (ICC $=0.88$ ) suggesting that this was not an anomaly.

What are the possible explanations for this bias? There is no doubt that many patients like to please their doctor and if they are given a new medication, they will report that they feel better. In addition, a clinician prescribing a medication of known therapeutic benefit will expect the patient to improve [5]. Recent evaluation of transition questions (i.e. those asking about change over time) has shown that they too can be very susceptible to bias [6]. If patients are feeling well today, they will have a tendency to say they feel better and if they are feeling ill today, they will say they feel worse. These responses are given independent of the initial state. Taken in conjunction with the first two well recognised biases, clinicians may have increased the shift further by basing their judgements of change more on the current status than on true change. Furthermore, if a patient had been under a particular clinician's care for some time, there may have been a tendency to remember a bad phase of the patient's history rather than the status at the previous visit when answering the transition question. It would be wrong not to recognise that the bias may have occurred the other way round with clinicians being right and the ACQ wrong. However, this seems highly unlikely because not only has the ACQ demonstrated very strong measurement properties and evidence of validity, the items in the questionnaire are those used by clinicians to estimate asthma control.

An unexpected observation should always be followed by a formal study to test the hypothesis in a scientifically rigorous manner. However, it witl be extremely difficult to test the bias observed in this study because as soon as clinicians know that they are being tested for bias, they will probably alter their behaviour! Certainly further retrospective analyses of large clinical trial databases where clinicians have been asked to estimate the efficacy of trial interventions might be used to collaborate or refute our observation but prospective studies will be difficult.

Clinicians usually make treatment decisions concerning asthma control based on objective measurements of airway status, (e.g. airway calibre, sputum eosinophils, exhaled NO etc.) and patients' subjective reporting of their symptoms, sleep disturbance and activity limitations etc. They integrate this information with their own clinical experience to estimate patients' current asthma control. However, Boulet and colleagues have identified that $43 \%$ of inadequately controlled asthma patients were rated adequately or well-controlled by clinicians [7]. This study extends their observation and shows that clinicians are equally inaccurate at estimating change in asthma control.

In conclusion, the results of this study have important implications both for the management of individual patients in routine clinical practice and for clinical trials where clinicians' estimates of change are often considered an important outcome. Clinicians should be conscious of the bias that may occur when estimating change in asthma control and know that it is much more accurate to measure asthma control at each visit using a validated instrument, than to rely solely on clinical judgement. Similarly, pharmaceutical companies should be aware of the questionable validity of asking clinicians about the effect of interventions and endeavour not to use transition rating questionnaires in clinical trials.

\section{Acknowledgements}

Financial Support: AstraZeneca, Lund, Sweden.

\section{References}

[1] Juniper EF, Guyatt GH, Willan A, Griffith LE. Determining a minimal important change in a disease-specific quality of life jnstrument. J Clin Epidemiol 1994;47:81 7 .

[2] Juniper EF, O'Byrne PM, Guyatt GH, et al. Development and validation of a questionnaire to measure asthma control. Eur Respir J 1999; 14:902-7.

[3] Juniper EF, Ståhl E, O’Byrne PM. Minimal important difference for the asthma control questionnaire. Am J Respir Crit Care Med 2001;163:642 (A).

[4] Juniper EF, Ståhl E, Mörk A, Svensson K. Minimal important difference for the asthma control questionnaire. Eur Respir $\mathrm{J}$ (Submitted).

[5] Sackett DL. Bias in analytic research. J Chron Dis 1979; 32:51-63.

[6] Guyatt GH, Norman GR, Juniper EF, Griffith LE. A critical look at transition ratings. J Clin Epidemiol 2002;55: 900-8.

[7] Boulet L-P, Phillips R, O'Byrne P, Becker A. Evaluation of asthma control by physicians and patients: comparison with current guidelines. Can Respir J 2002;9:417-23.

Available online at www.sciencedirect.com

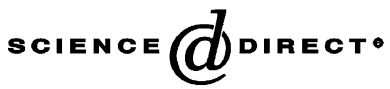

